\title{
Philosophiques
}

\section{Jean Grondin, Kant et le problème de la philosophie : l'A Priori, Librairie philosophique Vrin : Paris, 1989.}

\section{W. A. Shearson}

Volume 22, numéro 2, automne 1995

URI : https://id.erudit.org/iderudit/027356ar

DOI : https://doi.org/10.7202/027356ar

Aller au sommaire du numéro

Éditeur(s)

Société de philosophie du Québec

ISSN

0316-2923 (imprimé)

1492-1391 (numérique)

Découvrir la revue

Citer ce compte rendu

Shearson, W. A. (1995). Compte rendu de [Jean Grondin, Kant et le problème de la philosophie : l'A Priori, Librairie philosophique Vrin : Paris, 1989.]

Philosophiques, 22(2), 540-544. https://doi.org/10.7202/027356ar d'utilisation que vous pouvez consulter en ligne.

https://apropos.erudit.org/fr/usagers/politique-dutilisation/ 
Jean Grondin, Kant et le problème de la philosophie : l'A Priori, Librairie philosophique Vrin : Paris, 1989.

\section{par W. A. Shearson}

Grondin déclare son intention centrale dès la première phrase de cet ouvrage : «[...] de comprendre le sens de la Critique de la raison pure. » (p. 7) Comme nous allons le voir, non seulement a-t-il réussi à cet égard, mais son compte rendu de la Critique toute entière est exceptionnel. D'autres ont identifié des éléments très importants de l'œuuvre dans son ensemble, mais Grondin est le premier, à ma connaissance, à rassembler tous ces éléments et à nous donner, conséquemment, non seulement un compte rendu du problème - à vrai dire, des problèmes - de la première Critique de Kant, mais aussi à prendre au sérieux les dernières sections de cette ceuvre, ce qui permet d'éclaircir cette dernière encore plus et, ce qui est bien plus important, la relation systématique entre la première et la seconde Critique - la Critique de la raison pratique.

Après avoir déclaré son objectif général, Grondin identifie rapidement et correctement le problème central de la première Critique : «[...] la question d'une connaissance qui soit rigoureusement synthétique et a priori. Voilà le problème de la Critique de la raison pure.» (p. 7) Kant nous dit à trois reprises dans la Préface de la première édition que ceci est son problème (Axi, Axiv), que l'on peut résumer par cette déclaration définitive : « [...] la question capitale reste toujours de savoir : Que peuvent et jusqu'où peuvent connâ̂tre l'entendement et la raison, indépendamment de l'expérience. » (Axvii) ${ }^{1}$. Personne ne sera surpris d'apprendre que c'est là le problème de Kant, excepté que la plupart des savants semblent avoir ignoré ces déclarations.2.

1. Kant, Critique de la raison pure, trad.: A. Tremesaygues et B. Pacaud, Presses universitaires de France, Paris 1975, p. 9, Désormais cité CRP.

2. Voir Grondin, p. 7 : « Ce qui nous a incité à revenir sur cette question, c'est l'absence presque totale dans la littérature kantienne d'une étude qui réponde [...] aux réquisits du problème soulevé par Kant [...]» Aussi p. 51-52: « Il est plus que classique de présenter [...] à l'objet. » 
Grondin est tout aussi intelligent concernant la signification du problème. Premièrement, il comprend que la question de Kant porte sur ce qu'on peut connaître à propos des objets non-empiriquement. Cette connaissance doit être synthétique ou bien composée de jugements dans lesquels le concept du prédicat étend réellement le concept du sujet, car les jugements analytiques ne sont pas de véritables affirmations de connaissance. Deuxièmement, de tels jugements doivent être a priori et ainsi la question qu' on se pose pour en arriver à une véritable connaissance a priori est la même : "Comment des jugements synthétiques a priori sont-ils possibles ${ }^{1}$ ? »

Cependant, même cette formulation n'est pas adéquate, car selon Kant, nous possédons déjà au moins deux champs de connaissances dont les jugements sont synthétiques et a priori, soit l'arithmétique et la géométrie. Lorsque Kant demande, que peut-on connaître a priori, il veut dire, que peut-on connaître universellement et nécessairement (les deux aspects centraux de l'a priori) sur les objets-en-général. En un mot, les jugements a priori dont Kant nous parle sont métaphysiques. La question est donc identique à celleci : Comment la métaphysique comme science est-elle possible ? Pourquoi ? Parce que les jugements métaphysiques doivent être universel et nécessaire ; l'un parce que c'est une affirmation de connaissance véritable sur tout (objetsen-général) et l'autre parce que toute vérité sur tout objet possible serait nécessairement vraie. Donc, de tels jugements doivent être synthétiques et $a$ priori (l'empirique - l'a posteriori - ne cède jamais à la nécessité, selon Kant (A734/B762) ${ }^{2}$. De plus, de demander comment ces jugements sont possibles veut aussi dire vérifiables. Toute science doit pouvoir démontrer comment elle arrive à la véracité ou à la fausseté de ses jugements. La question de la métaphysique en tant que science est donc basée sur la vérification de ses jugements ${ }^{3}$.

Grondin comprend bien tout ceci. Cependant, il n'est pas aussi clair qu'il pourrait l'être pour expliquer pourquoi ce problème est un problème. Quel est le problème que nous présente la question de la vérification des jugements métaphysiques? Brièvement, toute vérification sert à établir la vérité d'une relation présumée entre le terme sujet et le terme prédicat dans le jugement. Dans le cas d'un jugement métaphysique synthétique a priori, la relation ne peut être purement logique, parce que le jugement est synthétique, et ne peut pas non plus être basé sur l'expérience, parce que le jugement est a priori.

1. CRP, p. 43 - sous le titre de section VI : Problème général de la raison pure.

2 CRP, p. 505.

3. Ceci veut dire qu'une des pires erreurs des études érudites sur Kant est de croire que la première Critique nous donne une épistémologie. Kant ne s'intéresse pas à la connaissance ; seulement à la connaissance synthétique a priori, qui est, de plus, métaphysique. Que Grondin observe ceci, voir p. 51-52 tel que cité préalablement au $n^{\circ} 2$, surtout que Kant souhaite « de résoudre le problème de la [...] connaissance a priori, pas célui de la connaissance en général. [...] 》 (j'ai ajouté les italiques). 
Ainsi, comme le dit Kant lui-même, si une telle relation n'est ni logique, ni empirique, que peut-elle être. Si je ne peux découvrir ceci, je ne pourrai jamais savoir comment vérifier ce jugement. Dans quelle sorte de jugement le concept du prédicat est-il joint au concept du sujet de façon non-logique (synthétique) et cependant avec nécessité (a priori), c'est-à-dire nonempiriquement ${ }^{1}$ ? Sa solution au problème est discutée dans toute la première partie de la première Critique, jusqu'à la Dialectique transcendentale (A293/B350).

Grondin explique tout ceci au Chapitre II intitulé La Critique transcendantale. Il passe ensuite à La Déduction transcendantale, que l'auteur appelle " la preuve de la révolution copernicienne au sujet de l'a priori", et c'est " là où se décide toute philosophie. " (p. 53) Encore une fois, Grondin nous démontre sa maîtrise du texte. En seulement vingt-cinq pages, il arrive à résumer avec précision l'essentiel de ce célèbre texte kantien mieux que plusieurs autres ne l'ont fait avec plus de détail et de pages ${ }^{2}$. Dans cette section, il confronte la question souvent oubliée (par les autres savants) et très peu évidente, de la distinction kantienne entre les jugements de perception et les jugements d'expérience, fournissant ainsi au lecteur un lien utile entre cette Critique et le Prolegomena. En ce qui concerne la Déduction, Grondin écrit : « L'idée d'une législation a priori de la nature par l'entremise d'une union de la sensibilité et l'entendement incarne le véritable terminus ad quem de la déduction [...] » (p. 71) À cet égard, mentionnons seulement que plus on comprend le problème, meilleures sont les chances d'en comprendre la solution.

Aussi exceptionnels et importants que soient ces éléments du livre de Grondin, le chapitre IV, Vers une métaphysique des intérêts de la raison, constitue la véritable contribution originale et profondément érudite qui rend cet ouvrage si important. C'est ici que nous apprenons quel est le but ultime de la solution à la question de l'a priori de la raison théorique ou spéculative. Cette solution nous dévoile de façon positive comment la métaphysique en tant que science est possible ; mais cette connaissance positive contient le but négatif des limites d'une telle connaissance. Ces limites absolues détournent la raison de son intérêt spéculatif (où elle ne peut être satisfaite), vers "l'unique voie qui lui reste encore, celle de l'usage pratique." (A796/B824) - ce que Kant appelle «le but final de l'usage pur de notre raison».

1. CRP, p. 39 (A9/B13). Il est à noter que ceci veut dire que l'Esthétique transcendantale ne se préoccupe pas de la possibilité des mathématiques, dont les fondements sont, selon Kant, prouvé entièrement par l'actualité de la science et ne nécessite aucun support philosophique. (Bxff) (CRP, p 16-17) Kant examine les jugements arithmétiques et géométriques parce qu'ils sont synthétiques et a priori et qu'aucun problème de vérification ne se pose. Il espère trouver là un indice qui l'aiderait avec la vérification métaphysique synthétique a priori. Cet indice est qu'il existe deux intuitions a priori, le temps et l'espace. (Voir Grondin, p. 49 ff.)

2. Á l'exception de D. P. Dryer, Kant's Solution For Verification in Metaphysics University of Toronto Press, Toronto, 1966. 
$(A 797 / B 825)^{1}$ Grondin voit clairement que tous les thèmes majeurs de l'ouvrage futur - l'utilisation pratique de la raison - sont déjà annoncés dans la première Critique. Il fait une distinction critique entre les deux utilisations de la raison, la théorique et la pratique, en réalisant parfaitement que l'intérêt de la raison envers la première est déplacée, car « le terrain qui est propre (à la raison), (est celui) des principes pratiques. " (A794/B822) ${ }^{2}$ Comme nous le dit Grondin : "La première et la plus indispensable fonction de la raison consiste à déterminer a priori l'agir des hommes. Condamnée à l'impuissance théorique, la raison ne peut être féconde que dans la sphère "pratique" [...]» (p. 87) D'autres savants (même Dryer) qui abordent le problème de l'a priori dans la première Critique, ne réussissent pas à démontrer que le problème de la possibilité d'un jugement synthétique a priori est uniquement le problème de l'a priori théorique ${ }^{3}$.

Donc, la vraie révolution dans la pensée de Kant est ce déplacement des grandes questions de la métaphysique - la liberté, Dieu, et l'immortalité de l'âme - du théorique vers le pratique. Comme le dit Grondin, c'est « un changement de paradigme dans l'histoire de la métaphysique.» (p. 93) et qu'il " (déclenche) une révolution dans notre intelligence des priorités philosophiques. » (p. 94) Encore une fois, ceci ne devrait surprendre personne, car Kant lui-même déclare que le but le plus important de la philosophie et de la métaphysique est celui de science des limites de la raison humaine ${ }^{4}$, et que ce qui est réellement important dans la philosophie c'est « la restauration des droits de l'homme" $"$.

Ce qui est le plus important dans l'ouvrage de Grondin, c'est qu'il nous donne le contexte de base pour comprendre la relation entre toutes ces affirmations, et ainsi le but systématique qui traverse toute la pensée de Kant, du début à la fin. Peu ont remarqué que la philosophie de Kant a pour but d'être un ensemble systématique. Dans le traitement qu'en a fait Grondin, il ne manque que la place de la troisieme Critique - la Critique du jugement - dans l'ensemble total. Il ne la mentionne qu'indirectement, ce qui est dommage. Si

1. $C R P$, p. 538 et 539 respectivement. Jusqu' ì maintenant, toute cette discussion concernant les problèmes de l'a priori métaphysique ne s'applique qu'à l'utilisation théorique de la raison. Ce qu'il faut comprendre avant tout c'est comment la solution au problème ouvre la porte à la possihilité d'une métaphysique de la raison pratique.

$2 \quad C R P$, P. 628

3. Une exception importante est Susan Shell, qui fut la première, en anglais du moins, à reconnaître non seulement l'importance du pratique chez Kant, mais sa primauté sur le théorique - la vraie révolution dans la pensée de Kant. Voir The Rights of Reason, University of Toronto Press, Toronto, 1980, dans lequel elle voit que pour Kant, la raison n'a non seulement des intérêts mais des droits, que l'on retrouvent tous dans le domaine du pratique. (Shell est théoricienne politique et s'intéresse surtout à la doctrine du Droit chez Kant.)

4. Bemerkungen, XX 181, Cf. aussi CRP, p. 505.

5. Ibid., $\mathrm{XX} 44$. 
toutefois on examine cette cuvre avec le cadre grondinien, on sera surpris d'apprendre que ce n'est pas principalement un ouvrage sur l'esthétique une erreur à laquelle même Grondin semble succomber (voir p. 106).

Le titre de ce livre laisse entendre que tout l'ouvrage porte sur Kant. Ce n'est pas le cas, car environ le dernier tiers de l'ouvrage s'intitule De l'A Priori après Kant. En à peu près soixante-dix pages, Grondin tente de traiter de l'a priori chez Reinhold, Schulze, Fichte, Schelling, Hegel, Cohen, Husserl, Heidegger, Gadamer et Habermas. Il vaut mieux ne pas trop en dire concernant ces pages. Je m'en tiendrai à trois brefs commentaires. Premièrement, on se demande pourquoi un savant qui venait juste d'écrire si brillamment concernant un philosophe, tenterait de nous entretenir sur dix autres philosophes en soixante-dix pages! Deuxièmement, on se demande encore plus pourquoi un savant prétendrait connaître suffisamment la pensée de dix philosophes, en plus de Kant, pour tenter de mettre ses idées sur papier. Finalement, deux des philosophes en question sont au moins aussi importants que Kant, soit Hegel et Heidegger, et de bien des manières, ils sont plus difficiles. L'effort que fait Grondin pour traiter de Hegel par exemple, en neuf pages et demi, est tout simplement grotesque. De plus, l'esquisse qu'il nous en fait suffit pour démontrer une erreur fondamentale, et donc, étant donné l'importance de Hegel, une incompréhension du destin de l'a priori kantien dans un monde post-Kant mortuum.

Le traitement de Kant par Grondin est exceptionel dans son ensemble. Aucune étude érudite sur Kant ne pourra désormais ignorer cet ouvrage. Il est très bref, claire et net, et en tant que tel, il prépare une base essentielle sur laquelle le savant prudent pourra poser une étude plus délaillé. Toutefois, Grondin a réellement écrit deux livres ici, le premier, comme l'indique le titre ; et le malheureux " postscript " à l'ouvrage sur Kant. La Conclusion aurait dû débuter à la fin du Chapitre IV - la section qui termine le traitement de Kant.

Département de philosophie

Bishop University

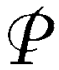

